TU1D-5

\title{
A MONOLITHIC HEMT DIODE BALANCED MIXER FOR 100-140 GHZ
}

\author{
Matthew Morgan ${ }^{1}$ and Sander Weinreb ${ }^{2}$
}

${ }^{1}$ Department of Electrical Engineering, California Institute of Technology, Pasadena, CA. 91125

${ }^{2}$ Jet Propulsion Laboratory, California Institute of Technology, Pasadena, CA. 91109

\begin{abstract}
We report the design and evaluation of a broadband, balanced mixer for 100-140 GHz using a HEMT MMIC process on a $75 \mu \mathrm{m}$ InP substrate. The circuit uses the gate Schottky diodes as mixing elements. It demonstrates a conversion loss of $15 \pm 2 \mathrm{~dB}$ from 100-130 GHz with $5 \mathrm{dBm}$ LO drive at $80 \mathrm{GHz}$. Measurements indicate a wide IF bandwidth extending beyond $50 \mathrm{GHz}$. This is the first demonstration of a monolithic HEMT diode balanced mixer in this frequency range.
\end{abstract}

\section{INTRODUCTION}

Broadband mixers are important components for millimeter-wave radiometers. A monolithic approach enables compact receivers to be assembled into a focal plane array for millimeter-wave imaging applications. HEMT diodes have been used in recent MMIC mixers [2][4] with encouraging results. This approach has the advantage of being compatible. with existing LNA technology, allowing a complete millimeter-wave downconverter to be integrated monolithically [3].

Balanced mixers have the useful property of suppressing LO amplitude noise as well as some high order mixing products [1]. Unfortunately, the bandwidth of monolithic balanced mixers is limited by that of the RF/LO coupler, which must have $3 \mathrm{~dB}$ coupling and $180^{\circ}$ output phase difference across the band to realize these benefits $\left(90^{\circ}\right.$ couplers may also be used, but suffer from poor RF-to-LO isolation). For example, the branch-line or ring hybrids are common choices for microstrip balanced mixers, but the bandwidth is typically only $10 \%$ to $20 \%$ [6].

This paper presents the design and evaluation of a broadband balanced diode mixer with RF from 100-140 $\mathrm{GHz}$, and IF from $20-60 \mathrm{GHz}$. The circuit is fabricated on InP using a standard HEMT MMIC process.

\section{CIRCUIT DESIGN}

The circuit is designed to downconvert an RF band of $100-140 \mathrm{GHz}$ to an IF of $20-60 \mathrm{GHz}$ by mixing with a fixed $80 \mathrm{GHz}$ LO. A photograph of the chip is shown in Figure 1 and a schematic is in Figure 2. As described earlier, the $180^{\circ}$ coupler is a key component of this type of

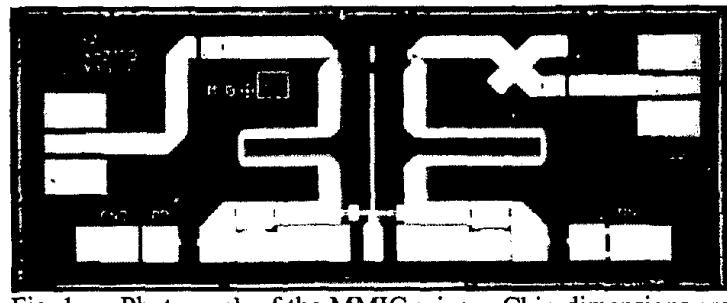

Fig. 1. Photograph of the MMIC mixer. Chip dimensions are $2.0 \times 0.74 \mathrm{~mm}$. InP substrate thickness is $75 \mu \mathrm{m}$.

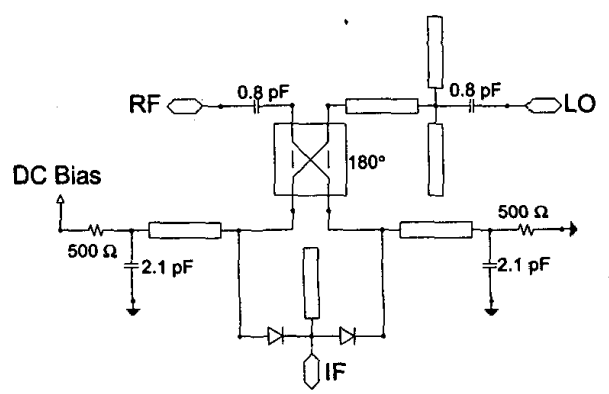

Fig. 2. Schematic of the mixer, showing MIM capacitors, thin-film resistors, and transmission line sections.

mixer, and constrains the bandwidth in many implementations. To overcome this limitation, we incorporate the novel combination of a Lange coupler with $90^{\circ}$ coupled-line phase shifters [7]-[8] to synthesize a broadband $180^{\circ}$ coupler, as shown in Figure 3. The Lange coupler was fabricated in the First-Level Interconnect (FIC) metal layer, which is thinner and allows tighter spacing between lines than top-metal in this process. Although this approach incurs greater losses, it was necessary to achieve the tight $3 \mathrm{~dB}$ coupling that is critical to the operation of the mixer. The coupled-line phase shifters that follow are designed to provide good input match and a differential phase shift of $90^{\circ} \pm 5^{\circ}$ from 80 $140 \mathrm{GHz}$. Both the coupler and the phase shifters were simulated using the HP Momentum software. Together they comprise a $180^{\circ}, 3-\mathrm{dB}$ coupler with approximately $55 \%$ bandwidth. 


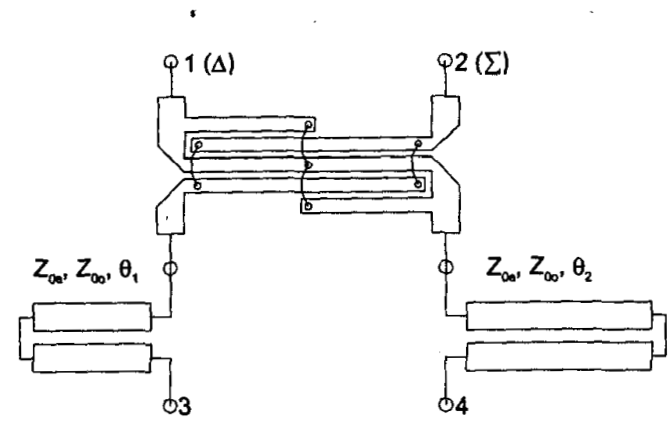

Fig. 3. Schematic of the $180^{\circ}$ directional coupler.

Two HEMT-gate Schottky diodes, available in the TRW InP HEMT Process [9], were used as mixing elements. Based on a 2-finger, $40 \mu \mathrm{m}$ FET model for this process, the gate structure was designed to simultaneously minimize the parasitic resistance and capacitance of the Schottky diode. Specifically, the diode cell consists of two fingers, each $5 \mu \mathrm{m}$ long. Our conservative estimate is that this structure has $R_{s}=33 \Omega$, and $C_{j 0}=16 \mathrm{fF}$. More rigorous testing of this structure leading to a better model of the diode is planned for future wafer runs.

The diodes are biased in series through low-impedance transmission lines along the bottom edge of the chip. The lines are bypassed at a quarter-wavelength from the diode in the center of the RF band. This provides a ground return and isolation for the IF, while allowing RF and LO frequencies to pass.

Directly on the IF port, between the two diodes, is an open-circuited stub which is intended to be a quarterwavelength long at the LO frequency. This was done to prevent LO power from leaking at this port. The stub was designed to be tunable by including a series of air-bridges - near the open end, which could be selectively broken to shorten the stub's length.

A microstrip matching section tuned for $80 \mathrm{GHz}$ was added to the LO side. No matching section was used on the RF port, because this point is too far from the diodes to match the signal over such a wide bandwidth. Nevertheless, simulations indicate that the input return loss should be greater than $6 \mathrm{~dB}$ across the band.

\section{MEASUREMENTS}

The test setup is shown in Figure 4. A Thomson Components Backward Wave Oscillator (BWO), model RWO $110 \mathrm{~S}$, was used to provide the LO signal. The power from the BWO was adjusted with a WR-10

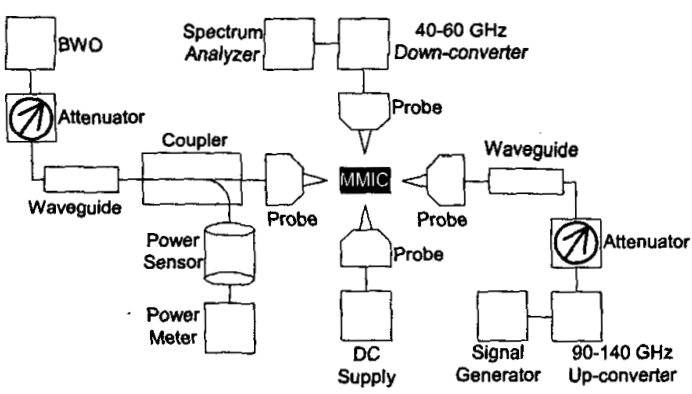

Fig. 4. Block diagram of the test setup. The $40-60 \mathrm{GHz}$ down-converter was omitted for IF $<40 \mathrm{GHz}$.

precision attenuator and monitored through a $10 \mathrm{~dB}$ coupler with an HP W8486A power meter. The LO power was coupled into the MMIC through a GGB Industries coplanar wafer probe. The precise coupling of the coupler, always close to $10 \mathrm{~dB}$, was recorded and used in the calibration of the measurements. The insertion loss of the wafer probe, about $1.3 \mathrm{~dB}$, was also calibrated out of the measurement.

The RF signal was supplied by an HP $83650 \mathrm{~B}$ Signal Generator, feeding a Millitech $90-140 \mathrm{GHz}$ waveguide mixer utilized as an up-converter. A $120 \mathrm{GHz}$ low-pass filter was attached to the $90 \mathrm{GHz}$ second harmonic Gunn on the Millitech module to remove a strong spurious tone at $135 \mathrm{GHz}$. RF signal power was adjusted with a WR-8 precision attenuator. Finally, the RF signal was coupled into the chip through another GGB Industries wafer probe. The attenuator setting that provided $-15 \mathrm{dBm}$ of signal power at the probe tips was measured and recorded for each frequency point. This signal level was strong enough to allow easy measurement of conversion loss without entering compression.

The IF signal was extracted with a $50 \mathrm{GHz}$ wafer probe. For IF below $40 \mathrm{GHz}$, the output was measured directly with an HP 8564E Spectrum Analyzer. The loss in the coaxial cable, typically on the order of $2-8 \mathrm{~dB}$, was calibrated out. To extend the range of IF measurements, a 40-60 GHz down-converter module was added in front of the Spectrum Analyzer, as shown in Figure 4. The conversion loss of this module is about $14-18 \mathrm{~dB}$, and was calibrated out.

The most significant results are plotted in Figures 5-8. As shown in Figure 5, the conversion loss is about $15 \mathrm{~dB}$ overall from $100 \mathrm{GHz}$ to $130 \mathrm{GHz}$. Although the circuit was designed for an $80 \mathrm{GHz} \mathrm{LO}$, additional data was taken with other LO frequencies in order to get some idea how the conversion loss changes with RF and IF frequencies 


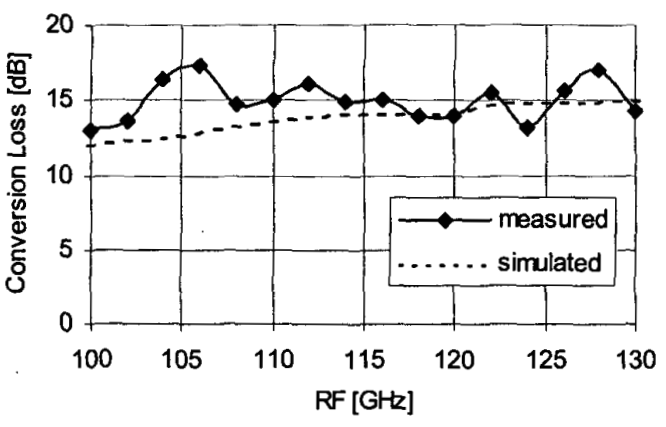

Fig. 5. Measured and simulated conversion loss vs. RF frequency. LO was $+5 \mathrm{dBm}$ at $80 \mathrm{GHz}$. RF power was -15 $\mathrm{dBm}$. The circuit was biased with a constant current of $2.4 \mathrm{~mA}$.

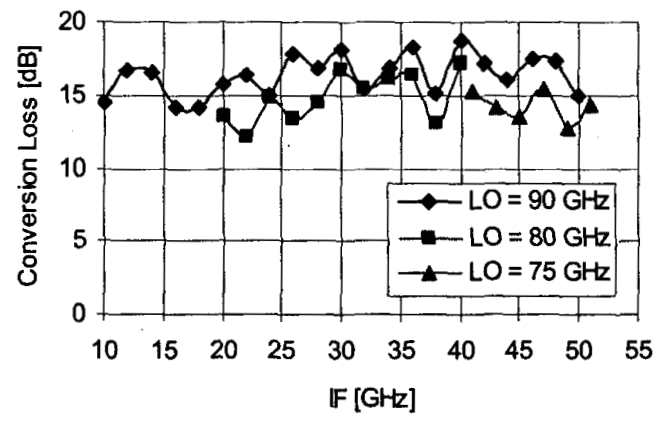

Fig. 6. Measured conversion loss vs. IF frequency for various LO frequencies. LO power was $+5 \mathrm{dBm}$ in all cases. RF power was $-15 \mathrm{dBm}$, and the bias was held constant at $3.6 \mathrm{~V}$.

that would otherwise be outside the range of the measurement setup. The results are shown in Figure 6. The conversion loss remains in the vicinity of $15 \mathrm{~dB}$ for $\mathrm{IF}$ from $10-50 \mathrm{GHz}$, and RF from $100-135 \mathrm{GHz}$.

The conversion loss in the center of the band for various LO powers and bias conditions is mapped out in Figure 7. Each curve represents a different LO power, and illustrates a different optimum current level. The curves converge at the low current end, implying that the conversion loss can be made relatively independent of $L O$ power if a sacrifice of a few $\mathrm{dB}$ in conversion loss is acceptable.

The amount of LO power the circuit can withstand and how it affects the conversion loss would be of interest in some applications. As shown in Figure 8, the LO power was swept up to $17 \mathrm{dBm}$, limited by the test equipment, without any detectable damage to the chip. A minimum of $12 \mathrm{~dB}$ conversion loss was found with $10 \mathrm{dBm}$ LO power, though one would expect the noise performance of the mixer to suffer at this drive level.

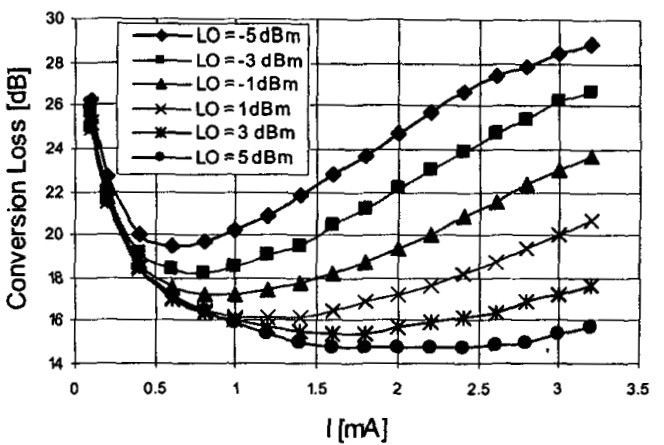

Fig. 7. Conversion loss vs. LO power and bias condition. The LO frequency was $80 \mathrm{GHz}$, and $\mathrm{RF}$ was $-15 \mathrm{dBm}$ at $110 \mathrm{GHz}$.

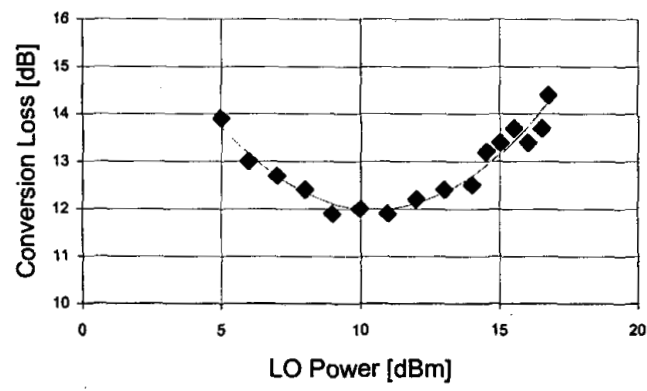

Fig. 8. Conversion loss vs. LO power for high drive level. The LO frequency was $88 \mathrm{GHz}$, and the RF signal was $-15 \mathrm{dBm}$ at $132 \mathrm{GHz}$. Bias was held constant at $2.4 \mathrm{~mA}$.

\section{CONCLUSION}

A broadband, balanced mixer for $100-140 \mathrm{GHz}$ has been demonstrated using a monolithic HEMT process. The chip demonstrates between 13 and $17 \mathrm{~dB}$ conversion loss from 100-135 GHz. The design incorporates a novel $180^{\circ}$ microstrip coupler with $55 \%$ bandwidth, which enables the broadband balanced mixer. This is the first monolithic HEMT diode balanced mixer in this frequency range.

\section{ACKNOWLEDGEMENT}

The authors wish to express their thanks to the staff of TRW for fabrication of the chip, to Douglas Dawson of JPL for invaluable laboratory assistance, and to Scott Kee, Ichiro Aoki, and Professor David Rutledge of Caltech for advice and discussion. The research described in this paper was carried out in part by the Jet Propulsion Laboratory, California Institute of Technology, under 
contract with the National Aeronautics and Space Administration through support of the Cross-Enterprise Technology Development and Instrument Incubator Programs.

\section{REFERENCES}

[1] S. Maas, Microwave Mixers, $2^{\text {nd }}$ ed. Boston, MA: Artech House, 1993.

[2] Y. L. Kok, H. Wang, M. Barsky, R. Lai, M. Sholley, and B. Allen, "A 180-GHz monolithic sub-harmonic InP-based HEMT diode mixer," IEEE Microwave Guided Wave Lett., vol. 9, pp. 529-531, December 1999.

[3] K. Chang, H. Wang, K. Tan, S. Bui, T. Chen, G. Dow, J. Berenz, T. Ton, D. Garske, T. Lin, and L. Liu, "A W-Band monolithic downconverter," IEEE Trans. Microwave Theory and Tech., vol. 39, pp. 1972-1979, December 1991.

[4] Y. Kok, P. Huang, H. Wang, B. Allen, R. Lai, M. Sholley, T. Gaier, and I. Mehdi, " 120 and $60 \mathrm{GHz}$ monolithic InPbased HEMT diode sub-harmonic mixer," IEEE MTT-S Intl. Microwave Symp. Digest, pp. 1723-1726, Baltimore, MD, 1998.

[5] W. Menzel, "A $140-\mathrm{GHz}$ balanced mixer for finline integrated-circuits," Microwaves \& RF, p. 34, 1983.

[6] R. Tahim, K. Chang, and K. Hummer, "Wideband microstrip balanced mixer," Electronics Letters, vol. 23, pp. 767-768, July 1987.

[7] Y. C. Leong and S. Weinreb, "Synthesis of Broadband TEM Coupled Line Phase Shifters," submitted.

[8] Y. C. Leong and S. Weinreb, "Novel technique of phase velocity equalization for microstrip coupled-line phase shifters," IEEE MTT-S Intl. Microwave Symp. Digest, pp. 1453-1456, Boston, MA, 2000.

[9] R. Lai, M. Barsky, T. Huang, M. Sholley, H. Wang, Y. Kok, D. Streit, T. Block, P. Liu, T. Gaier, and L. Samoska "An InP HEMT MMIC LNA with 7.2-dB gain at 190 GHz," IEEE Microwave Guided Wave Lett., vol. 8, pp. 393395, Nov. 1998. 